\title{
Acordos de empréstimo para AIDS e a Política de Incentivo no Sistema Único de Saúde: avanços e lacunas
}

\section{Loan agreements for AIDS and the National Health System Incentive Policy: advances and gaps}

\author{
Renato Barboza ${ }^{1,2}$ (D) \\ 'Universidade de São Paulo (USP) - São Paulo (SP), Brasil. \\ ${ }^{2}$ Instituto de Saúde - São Paulo (SP), Brasil.
}

Como citar: Barboza R. Acordos de empréstimo para AIDS e a Política de Incentivo no Sistema Único de Saúde: avanços e lacunas. Cad Saúde Colet, 2021;29(1):46-53. https://doi.org/10.1590/1414-462X202129010346

\section{Resumo}

Introdução: No Brasil, os acordos de empréstimo e a Política de Incentivo foram fundamentais para a gestão descentralizada da política de aids, entretanto poucos estudos avaliaram os seus efeitos no âmbito do Sistema Único de Saúde (SUS). Objetivo: Analisar a implementação das ações programáticas em DST/Aids no país e no estado de São Paulo quanto aos avanços e lacunas dos acordos de empréstimo com o Banco Mundial e a implantação da Política de Incentivo vigente no SUS. Método: Estudo de caso, retrospectivo-descritivo, baseado em análise documental e entrevistas em profundidade com seis coordenadores das três esferas governamentais e dois presidentes do Fórum Estadual de ONG/AIDS de São Paulo. Resultados: Identificaram-se diferentes graus e distintas formas de operacionalização da política de aids, demarcadas por períodos de centralização das decisões e do financiamento no "Projeto AIDS I", seguida pela desconcentração administrativa no final do "Projeto AIDS II"e, finalmente a descentralização com a Política de Incentivo, transferindo responsabilidades e recursos em consonância aos princípios do SUS. Conclusão: Não obstante os avanços da gestão descentralizada, fragilidades constatadas no processo de monitoramento e avaliação das ações podem comprometer a sustentabilidade técnicofinanceira da Política de Incentivo no SUS.

Palavras-chave: história; Banco Mundial; descentralização; desenvolvimento de programas; síndrome da imunodeficiência adquirida.

\begin{abstract}
Background: In Brazil, loan agreements and the Incentive Policy were fundamental to decentralize the management of the AIDS policy; however, few studies have evaluated their effects within the scope of the National Health System (NHS). Objective: To analyze the implementation of programmatic actions related to STD/AIDS in the country and in the state of São Paulo regarding the advances and gaps of the loan agreements with the World Bank and the implementation of the NHS Incentive Policy currently in force. Method: A retrospective descriptive case study based on documentary analysis and in-depth interviews with six coordinators from three governmental levels and two presidents of the State Forum for NGO/AIDS in São Paulo. Results: Different degrees and forms of operationalization of the AIDS policy were identified; they are marked by periods of centralization of decisions and funding in the "AIDS Project I", followed by administrative decentralization at the end of the "AIDS Project II" and, finally,
\end{abstract}

Trabalho realizado no Programa de Pós-Graduação em Ciências da Coordenadoria de Controle de Doenças da Secretaria de Estado da Saúde de São Paulo (PPG-CCD-SES/SP) - São Paulo (SP), Brasil.

Correspondência: Renato Barboza.E-mail: renato@isaude.sp.gov.br; renbarprof@gmail.com

Fonte de financiamento: nenhuma.

Conflito de interesses: nada a declarar.

Recebido em: Set. 25, 2017. Aprovado em: Jan. 06, 2020
Este é um artigo publicado em acesso aberto (Open Access) sob a licença Creative Commons Attribution, que permite uso, distribuição e reprodução em qualquer meio, sem restrições desde que o trabalho original seja corretamente citado. 
decentralization brought about by the Incentive Policy, which transfers responsibilities and resources in line with NHS' principles. Conclusion: Despite the progress of decentralized management, weaknesses along the process of monitoring and evaluation of actions may compromise the technical and financial sustainability of the NHS Incentive Policy.

Keywords: history; World Bank; decentralization; program development; acquired immunodeficiency syndrome.

\section{INTRODUÇÃO}

Na década de 1990, a aids foi incluída na agenda internacional como uma questão estratégica para o desenvolvimento social. Em 1997, o Banco Mundial disponibilizou US\$ 552 milhões em empréstimos para prevenção do HIV em 41 países'. No Brasil, a estratégia do Banco para o setor saúde priorizou três áreas: a ampliação do acesso na Atenção Básica; a oferta de conselhos políticos e estudos sobre a eficiência do sistema de saúde; e o controle de doenças transmissíveis, como a aids². Entre 2000 e 2015, o Banco financiou 32 projetos, sendo 28,2\% sob gestão da União e $71,8 \%$ de estados e municípios, privilegiando, portanto, a gestão descentralizada, diferentemente da década passada ${ }^{3}$.

No governo do presidente Itamar Franco (1992-1995) iniciaram-se as negociações entre o Ministério da Saúde e o Banco Mundial para o primeiro acordo de empréstimo bilateral para aids. Esse processo mobilizou uma ampla rede de atores internos e externos ao setor de saúde, sobretudo do Programa Nacional DST/Aids (atual Departamento de Vigilância, Prevenção e Controle de IST, HIV/Aids e Hepatites Virais), de Organizações Não Governamentais (ONGs), pesquisadores e docentes de universidades, entre outros $s^{4,5}$.

O acordo de empréstimo, implantado em 1994, com vigência de quatro anos ("Projeto AIDSI") compreendeu um aporte de US\$250 milhões, sendo US\$160 milhões do Banco e US\$90 milhões de contrapartida nacional, envolvendo $60 \%$ de recursos dos estados e municípios e $40 \%$ da União. Objetivou reduzir a incidência das DST/Aids e fortalecer as instituições públicas e privadas (ONGs), abrangendo 27 secretarias estaduais e 150 municípios estratégicos no controle da epidemia. No presente estudo, selecionamos o estado de São Paulo como cenário de investigação, por ser a unidade da federação que recebeu o maior volume de recursos e municípios conveniados no país (37 municípios), além da Secretaria Estadual de Saúde ${ }^{6}$.

Posteriormente foi financiado o "Projeto AIDS II", entre 1998 e 2003, com aporte de US\$296,5 milhões. Além da redução da incidência das DST/Aids, objetivou ampliar e qualificar o acesso ao diagnóstico e à atenção às pessoas vivendo com HIV/Aids (PVHA), bem como a sustentabilidade técnico-financeira das ações programáticas nos estados e municípios. O"Projeto AIDS III", executado entre 2003 e 2006 no valor de US\$ 200 milhões, visou à promoção da melhoria da qualidade de vida das PVHA e maior eficiência das ações na perspectiva da sustentabilidade ${ }^{7}$. A implementação desses projetos reorganizou sobremaneira o processo de trabalho das instituições governamentais e da sociedade civil engajadas no controle da aids no país ${ }^{6}$.

Em 2002, o Ministério da Saúde instituiu a Política de Incentivo para DST/Aids, com recursos oriundos do Sistema Único de Saúde (SUS) na modalidade fundo a fundo, transferindo recursos do Fundo Nacional de Saúde para os fundos estaduais e municipais, baseado na programação anual de ações e metas pactuadas pelos gestores ${ }^{8}$. São Paulo habilitou 144 municípios e a secretaria estadual, abrangendo $94,2 \%$ dos casos de aids notificados e municípios com mais de 70 mil habitantes ${ }^{9-11}$. Atualmente, o governo federal investe anualmente $\mathrm{R} \$ 129$ milhões no financiamento de ações de prevenção, vigilância epidemiológica e assistência às DST/Aids e hepatites virais em 26 estados, no Distrito Federal, além de 489 municípios, incluindo 10\% para projetos de ONGs, sob gestão estadual, abrangendo $62 \%$ da população brasileira e $89 \%$ dos casos notificados ${ }^{12}$.

No Brasil, a epidemia concentrada em algumas populações reforça a urgência de ações potentes para intervir nos contextos de vulnerabilidade, sobretudo entre usuários de drogas, homens que fazem sexo com homens e profissionais do sexo, segmentos que apresentam altas taxas de prevalência do HIV, comparados à população em geral ${ }^{13}$. Contudo, o país alcançou reconhecimento internacional e possui um dos melhores programas de aids estruturados entre os países em desenvolvimento ${ }^{14}$. Assim, é fundamental investigar a implementação das ações 
programáticas no país e no estado de São Paulo quanto aos avanços e lacunas dos acordos de empréstimo com o Banco Mundial e a implantação da Política de Incentivo vigente no SUS.

\section{MÉTODO}

No período entre 1994 e 2006 foram implementados três acordos de empréstimo com o Banco Mundial, os quais conformam um cenário privilegiado para investigar a resposta brasileira no enfrentamento da aids.

Realizamos um estudo de caso retrospectivo-descritivo de abordagem qualitativa ${ }^{15}$, com foco na análise retrospectiva do processo de negociação e implementação do primeiro e segundo acordos de empréstimo (1994 a 2003); nos resultados da gestão descentralizada no estado de São Paulo e em quatro municípios paulistas, definidos como cenário do estudo; e na implantação em 2002 da Política de Incentivo em curso no SUS. Conduzimos oito entrevistas semiestruturadas com informantes-chave que atuaram nesse período, sendo seis coordenadores de programas de DST/Aids das esferas nacional (1), estadual (1) e municipal (4) e dois presidentes do Fórum Estadual de ONG/Aids de São Paulo, baseadas em um roteiro previamente elaborado. Os critérios de seleção dos informantes foram fundamentados na experiência acumulada na implementação de políticas de aids no âmbito governamental ou da sociedade civil e no tempo superior a dois anos em posições de liderança. Segundo o critério de saturação dos dados, o número de participantes foi suficiente, permitindo apreender o objeto investigado ${ }^{16}$. Intencionalmente o estudo de caso foi aprofundado em quatro municípios de grande porte, com sistemas de saúde complexos e epidemiologicamente relevantes, a saber: Guarulhos, Campinas, São José dos Campos e Ribeirão Preto ${ }^{15,17}$. As entrevistas conduzidas em 2006 foram gravadas, transcritas e submetidas à análise de conteúdo para apreensão dos significados das narrativas, compreendendo as fases de pré-análise, exploração do material, tratamento dos resultados e interpretação ${ }^{18,19}$. O perfil dos entrevistados revelou vasta experiência profissional e participação ativa em colegiados e comitês técnico-políticos responsáveis pela condução da política de DST/Aids no SUS (Tabela 1).

Adicionalmente, conduzimos uma pesquisa documental baseada na identificação, catalogação das fontes primárias e análise dos documentos de gestão, identificados diretamente nas instituições ou por meio de consultas em sites e portais eletrônicos. Analisaram-se documentos referentes aos dois acordos de empréstimo, ao planejamento estratégico e aos planos operativos anuais dos programas estadual de DST/Aids São Paulo e dos municípios selecionados. O projeto foi aprovado pelo Comitê de Ética do Instituto de Saúde (parecer nº14/05) e os participantes entrevistados após a anuência do termo de consentimento.

Considerando-se a exígua produção científica sobre a avaliação da gestão descentralizada da política de aids no país e no estado de São Paulo, buscou-se conhecer retrospectivamente os principais aspectos técnico-políticos da execução dos dois empréstimos com o Banco Mundial e a transição para a "Política de Incentivo" em curso, financiada integralmente pelo SUS. Analisar esse percurso permitiu compreender as diferentes fases da gestão dessa política pública, sobretudo em São Paulo, que recebeu o maior montante de recursos. Assim, no campo da

Tabela 1. Perfil dos entrevistados, segundo tipo de liderança, gênero, tempo de trabalho em aids e participação em conselhos e comitês

\begin{tabular}{lcccc}
$\begin{array}{c}\text { Posição de liderança/ } \\
\text { No de informantes }\end{array}$ & Gênero & $\begin{array}{c}\text { Anos de } \\
\text { trabalho na } \\
\text { aids }\end{array}$ & $\begin{array}{c}\text { Participação } \\
\text { em Conselho } \\
\text { de Saúde }\end{array}$ & $\begin{array}{c}\text { Participação } \\
\text { em Comitê } \\
\text { Técnico/aids }\end{array}$ \\
\hline Coordenador nacional (1) & Masc. & 4 & Não & Sim \\
\hline Coordenador estadual (1) & Fem. & 12 & Sim & Sim \\
\hline Coordenador municipal (4) & Fem. & 6 a 10 & Sim (2) & Sim (2) \\
\hline Presidente Fórum de ONG/Aids (2) & Masc. & 4 a 6 & $\begin{array}{c}\text { Sim (1) } \\
\text { Não (1) }\end{array}$ & Sim \\
\hline
\end{tabular}


saúde coletiva, ressaltamos a função estratégica desempenhada por uma política ou programa no que concerne aos arranjos institucionais conduzidos pelos gestores, com o fito de alcançar os objetivos propostos ${ }^{20}$.

\section{RESULTADOSE DISCUSSÃO}

\section{Negociações com o Banco Mundial}

O acesso ao financiamento do Banco Mundial implica aderir às regras dessa agência internacional. Quando as posições são discordantes, a equipe do Banco investe em processos de negociação e oferta de ideias ${ }^{21}$. No que concerne ao primeiro acordo de empréstimo, os trâmites foram finalizados com celeridade, demonstrando a vontade política do governo brasileiro em relação à aids ${ }^{6}$. Corroborando esses estudos, um informante enfatizou que, "[...] durante as reuniões no Ministério da Saúde, a missão do banco e as equipes técnicas, incluindo o gabinete do ministro Adib Jatene, identificaram uma pauta comum que permitiu iniciar a cooperação" (E1). Cabe frisar que os acordos firmados posicionaram o país numa agenda inovadora e internacional, sendo um dos principais repasses concedidos para aids, vinculado à alocação de recursos do tesouro nacional ${ }^{22}$.

Um ponto de inflexão na relação entre o Brasil e o Banco Mundial, mesmo antes do empréstimo para aids, se referia aos gastos crescentes com a atenção integral e o acesso universal aos cuidados em saúde preconizados pelo SUS. Na visão do Banco, "[...] ocorrerão crescimentos explosivos na demanda de serviços à medida que aumenta a idade da população e que é exercido o direito constitucional de tratamento gratuito para todos ${ }^{\prime 23: 8}$, revelando assim concepções diferenciadas quanto ao papel do Estado no sistema de proteção social aos cidadãos. Entretanto, o enfrentamento da aids no Brasil data de 1983, portanto anterior ao acordo de empréstimo e à implantação do SUS em 1990. Adicionalmente, o Ministério da Saúde sempre considerou o empréstimo como um recurso complementar aos investimentos do tesouro. No que tange à política de aids, o "AIDS I" foi estratégico para dar visibilidade ao modelo brasileiro, pois como afirmou um coordenador: "[...] disseminou os princípios da resposta brasileira que antecedem o Banco" (E1).

Na ótica do Banco Mundial, o investimento na promoção de práticas seguras, como a distribuição de preservativos, deveria ser a principal estratégia para conter a epidemia, em detrimento do financiamento da assistência, sobretudo o acesso gratuito aos antirretrovirais pelas PVHA. Durante o processo de negociação e construção de consensos, essa posição foi arduamente defendida pelo Banco, baseado na falta de eficácia e no baixo custo-benefício dessa estratégia para reduzir a incidência. Em que pese às diferenças de ordem técnica e principalmente política, a gestão do "Projeto AIDS I" foi avaliada pelo Banco como "muito satisfatória"24. Constatou-se que houve divergências técnicas, políticas e ideológicas em relação às propostas defendidas pelo Banco Mundial, sobretudo quanto à pertinência dos princípios do SUS. Entretanto, outro coordenador ponderou que "[...] o projeto brasileiro aprimorou a atuação do banco, contribuindo com um novo paradigma no financiamento de países em desenvolvimento" (E2).

Apesar dessas discordâncias, o Banco aprovou, em 1998, o"Projeto AIDS II", demonstrando que as estratégias de negociação e intensificação do diálogo político continuaram sendo o foco das missões técnicas. No que concerne à sustentabilidade técnico-financeira das ações programáticas, verificamos que o Banco mudou a sua posição política ao afirmar que a mesma

[...] seria promovida pelo forte suporte do governo em serviços preventivos e de tratamento para HIV/AIDS. [...] Com relação aos recursos que o governo está gastando na saúde, e mais particularmente, em medicamentos para tratar pacientes com AIDS (fora do projeto proposto), os custos de continuidade das atividades contempladas no projeto são muito baixos ${ }^{24: 47}$.

\section{Implementação dos "Projetos AIDS I e II"}

Um dos principais ganhos do "Projeto AIDS I" referiu-se ao fortalecimento do processo de trabalho e da estrutura do Programa Nacional, contribuindo, sobretudo, para articulação 
junto às secretarias estaduais e municipais de saúde e ONGs conveniadas ${ }^{22}$. Os conveniados eram denominados "executores das ações" e elaboravam Planos Operativos Anuais (POAs) em consonância aos objetivos aprovados pelo Banco Mundial. Na visão de coordenador estadual, o Ministério da Saúde assumiu o papel de financiador"[...] numa perspectiva centralizada e os estados, municípios e ONGs só incorporaram de fato a descentralização no final de 1990" (E2). Entretanto, essa posição foi modificada ao longo da implementação do "AIDS I" com o fortalecimento da gestão estadual e municipal, mas cabe ressaltar que a racionalidade empregada pelo gestor federal estava na contramão da descentralização preconizada pelo SUS ${ }^{25}$.

$\mathrm{Na}$ visão dos municípios, a centralização dos recursos oriundos dos POAs foi decisiva para ampliação e diversificação das modalidades de atenção especializada em DST/Aids, organizando, assim, o processo de trabalho das equipes multidisciplinares de referência para atender a demanda crescente dos novos casos de HIV/Aids. Nessa perspectiva, um coordenador avaliou que, no seu município, "[...] os recursos marcados do Banco foram um grande avanço para estruturar serviços de referência, o centro de testagem, a assistência domiciliar e o hospital-dia" (E4).

No entanto, o relatório de avaliação do Banco Mundial apontou diversas vulnerabilidades no desenvolvimento do "AIDS I", como o quadro exíguo de profissionais na unidade de avaliação do Programa Nacional, comprometendo o monitoramento e a supervisão dos convênios; a desarticulação das ações preventivas e assistenciais; e a falta de treinamento das equipes ${ }^{24}$. No que tange às lições aprendidas no "AIDS II", consta do relatório do Banco que foram incorporadas estratégias para fortalecer a gestão descentralizada, a intersetorialidade e o controle social'.

A proatividade das coordenações estaduais na condução do financiamento das ONGs, como observado em São Paulo, foi associada no "AIDS II" a um processo de desconcentração técnico-administrativa, entretanto avaliada pelo Banco Mundial como um ponto frágil em nível nacional, porque apenas oito estados assumiram essa função ${ }^{24}$. No "AIDS I", o papel do gestor estadual estava circunscrito à supervisão dos projetos das ONGs, predominando a desconcentração de poder da esfera federal, restrita aos trâmites técnico-burocráticos. $\mathrm{Na}$ percepção de um presidente do Fórum de ONG/Aids, esse processo deve ser aprimorado quanto à definição de responsabilidades porque os gestores "[...] não têm a mesma linguagem e a competência de cada instância ainda não foi totalmente descentralizada" (E7). Cabe ressaltar que as parcerias entre ONGs e municípios tornaram-se mais complexas e exigiram habilidades técnicas e, sobretudo, políticas para negociar as DST/Aids na agenda municipal de saúde. Nesse sentido, outro presidente vocalizou que "[...] no município é mais complexo porque a aids não é uma prioridade" (E8). É possível inferir dessas narrativas que, no SUS, o controle social deve ser uma estratégia ímpar para o aprimoramento da gestão descentralizada, entretanto os gestores e os membros das ONGs ainda precisam se apropriar dos mecanismos existentes.

Não obstante o investimento contínuo dos "Projetos AIDS I e II" na formação das equipes municipais de saúde, um entrevistado pondera que "[...] as ações preventivas foram pontuais e com impacto reduzido" (E4). Constatou-se que a prevenção foi delegada às ONGs, corroborado pela baixa proatividade das coordenações municipais. Nesse sentido, um coordenador tencionou que "[...] a grande dívida dos municípios é a prevenção, porque não é realizada" (E1), corroborado por pesquisadores que postulam que o movimento social priorizou, na década de 1990, a luta pelo acesso ao tratamento em detrimento da prevenção ${ }^{26}$. Apesar disso é preciso reconhecer que as ações preventivas implementadas no país foram pouco eficazes no enfrentamento das tendências epidemiológicas ${ }^{13}$. Em grande angular, assim como relatado pelos informantes, estudos evidenciaram desigualdades regionais na gestão desses convênios, revelando gradientes na implementação das tecnologias de prevenção e assistência, no financiamento das ONGs e na efetividade dos recursos alocados ${ }^{25}$.

Foi dada ênfase na programação das ações, prejudicando um processo de planejamento amplo e contextualizado as necessidades locais, reiterando assim o caráter normativo e centralizador do Ministério da Saúde. Mas, na percepção de um informante, "[...] contribuiu para diferenciar a resposta brasileira para aids, já que não há um planejamento tão sistematizado em outras áreas, mas a missão não foi discutida" (E1).

A discussão da missão poderia ter qualificado a gestão da política nacional de DST/Aids, entretanto não foi levada a cabo. O Ministério da Saúde não teve dificuldades para incorporar 
o marco lógico do Banco à rotina gerencial, porque os convênios de outros setores já seguiam essa matriz. Todavia, os coordenadores municipais enfrentaram dificuldades devido à limitada capacidade técnico-administrativa para planejar e prestar contas nas secretarias de saúde, relatando que houve "[...] uma enorme dificuldade administrativa para executar o recurso" (E3).

Verificou-se que os coordenadores nacional e estadual apoiaram os municípios na gerência dos POAs. No Programa Estadual, o planejamento foi aperfeiçoado com a formação dos gerentes e a produção de informações para qualificar as decisões, culminando na elaboração do planejamento estratégico e a definição da missão e dos eixos prioritários da política estadual, delineando assim as bases para a implantação da Política de Incentivo ${ }^{8,27}$.

\section{Política de Incentivo para DST/Aids}

Considerando o desempenho dos projetos anteriores, o Banco Mundial aprovou o "Projeto AIDS III", destacando que os" [...] resultados estão entre os melhores" e que "[...] as lições aprendidas serão aplicáveis à maioria dos programas de prevenção apoiados pelo banco"7:5. O foco foi a sustentabilidade das ações atrelada ao fortalecimento da descentralização dos recursos financeiros, oriundos de fontes nacionais do SUS. Segundo relatório do Banco, a Política de Incentivo foi uma estratégia inovadora para assegurar a descentralização e a continuidade do financiamento nos estados e municípios ${ }^{7}$.

Os convênios ficaram obsoletos e alvo de críticas dos gestores em várias áreas da saúde. Em menos de uma década, o mecanismo convenial das DST/Aids devido à burocracia das regras do Banco, nem sempre compatíveis com a legislação da administração pública, levou à sua substituição ${ }^{28}$. A possibilidade de financiamento regular via fundo a fundo aliada à preocupação crescente com a sustentabilidade técnico-financeira foram os principais elementos que corroboraram essa decisão. Assim, gradualmente, o Programa Nacional adotou as regras do SUS utilizadas em outros programas, ratificadas por um dos coordenadores: "[...] a aids era uma das poucas áreas ainda com convênio" (E1).

Entretanto, para efetivar o incentivo, duas questões dependeram da pactuação nos colegiados. Uma questão era vincular o recurso às DST/Aids, o que implicou enfrentar resistências dos gestores. Ea outra, aumentar o investimento dos recursos próprios das secretarias para ampliar a cobertura das ações, como relatado por um informante: "[...] o maior problema era carimbar o dinheiro para um agravo e já tinha oposição dos secretários municipais criticando a indução de políticas pelo financiamento" (E1). Concordando com essa ponderação, um coordenador municipal foi categórico: "[...] se a verba não for exclusiva para aids, o programa acaba" (E4).

O instrumento de programação do pleito ao incentivo denominado "Plano de Ações e Metas" (PAM) está baseado no diagnóstico local e na avaliação dos avanços e desafios para o controle das DST/Aids nas áreas: promoção da saúde e prevenção, atenção às PVHA e portadores de outras DST, desenvolvimento institucional e parcerias com ONG, elemento obrigatório aos estados $^{8}$. Segundo definição da Comissão Intergestores Tripartite, devem ser alocados 25\% dos recursos próprios dos estados e municípios das regiões Sul e Sudeste e 15\% nas demais ${ }^{8}$. O PAM, atualmente denominado "Programação Anual de Saúde", depende da aprovação dos respectivos Conselhos de Saúde e Comissões Bipartite e Tripartite, sendo vinculado ao bloco de financiamento da vigilância em saúde ${ }^{11}$.

Em oposição aos convênios, a pactuação do incentivo nos colegiados do SUS foi ascendente e teve participação ativa dos gestores, e na opinião da coordenação estadual, "[...] não foi um pacote" (E2). Em São Paulo foi designada uma equipe assessora às coordenações municipais, posteriormente incorporada à estrutura do programa como gerência de planejamento. Nos municípios houve avanços no planejamento e implementação das ações, conferindo maior autonomia aos gestores, como relatado por um coordenador: "[...] o PAM permite rever as ações para alcançar as metas" (E3).

Não obstante os avanços, o monitoramento e a avaliação foram elencados pelos gestores como desafios. A observância do PAM e a produção de evidências para orientar as decisões foram sublinhadas como elementos essenciais à sustentabilidade das ações. No entanto, um informante identificou vulnerabilidades, demonstradas em outros estudos ${ }^{25,29}$, que podem comprometer os resultados dessa política, porque 
[...] os compromissos assumidos são executados de forma bem frágil, variam de região para região, e mesmo no Sudeste a capacidade de cumprir acordos é baixa, por vários motivos: amarração burocrática, ineficiência das estruturas, falta de prioridades e entendimento político dos secretários. (E3).

Pode-se inferir pela análise dos relatos e das fontes documentais que a racionalidade da Política de Incentivo contribuiu sobremaneira para o aprimoramento da gestão descentralizada das DST/Aids, alinhada às normas de financiamento vigentes no SUS ${ }^{10,11}$. Não obstante os avanços da descentralização na ampliação da oferta de serviços especializados, os recursos alocados ainda são incipientes e podem comprometer a efetividade das ações. Pesquisas sobre a descentralização revelam que as ações voltadas às populações mais vulneráveis foram exíguas e evidenciam que $93,6 \%$ das secretarias planejaram ações preventivas para população em geral, ao passo que no segmento dos homens que fazem sexo com homens apenas $21,4 \%$ das atividades, $34,8 \%$ para usuários de drogas e $44,5 \%$ para profissionais do sexo ${ }^{29}$. Corroborando esse cenário, fragilidades operacionais e políticas nas secretarias municipais ainda dificultam a aplicação adequada dos recursos, gerando saldo nas contas dos fundos. No entanto, desde 2013 o Ministério da Saúde preconiza a devolução dos valores não executados com correção monetária, com vistas a assegurar a eficiência da política de incentivo em curso no país ${ }^{11}$.

\section{CONCLUSÃO}

No que tange à gestão descentralizada, os achados demonstraram diferentes graus e distintas formas de operacionalização das ações programáticas nas secretarias estadual e municipais de saúde pesquisadas. Constatamos períodos de centralização das decisões e do financiamento no Ministério da Saúde, principalmente no "Projeto AIDS I", seguido pela desconcentração administrativa no final do "Projeto AIDS II" e, finalmente a descentralização após a implantação da Política de Incentivo, a qual transferiu responsabilidades e recursos financeiros aos gestores em consonância às diretrizes do SUS.

As evidências revelaram dificuldades operacionais, associada à limitada capacidade técnico-administrativa das coordenações estadual e, sobretudo, municipais. O planejamento foi restrito à programação de ações e prestação de contas ao Ministério da Saúde e ao Banco Mundial, evitando assim a descontinuidade dos repasses. Entretanto, essa lógica convenial foi alterada com a Política de Incentivo, conferindo autonomia aos gestores e o aperfeiçoamento da pactuação no SUS, ainda em vigência. Atualmente, os recursos alocados no bloco da vigilância em saúde reforçam a necessidade da pactuação da política de DST/Aids nas esferas loco-regionais ${ }^{10,11}$. Assim, como observado em outros estudos, as evidências demonstraram que a descentralização aumentou a complexidade da gerência das ações programáticas s $^{3,30}$. Nessa ótica, qualificar a dimensão técnico-política para a sustentabilidade das ações no SUS implica envidar esforços dos coordenadores para consolidar a Política de Incentivo, sobretudo quanto ao monitoramento e avaliação. Desta feita, recomenda-se o desenvolvimento de outros estudos sobre o impacto dessa política, especialmente no que concerne à redução da incidência do HIV nas populações mais vulneráveis.

\section{REFERÊNCIAS}

1. Dayton J. World Bank HIV/AIDS interventions: ex-ante and ex-post evaluation. Washington; 1998. (World Bank Discussion Paper; no. 389).

2. Banco Mundial. Relatório sobre o Desenvolvimento Mundial 1993: investindo em saúde. Rio de Janeiro: Fundação Getúlio Vargas; 1993.

3. Rizzotto MLF, Campos GWS. O Banco Mundial e o Sistema Único de Saúde brasileiro no início do século XXI. Saude Soc. 2016;25(2):263-76. http://dx.doi.org/10.1590/S0104-12902016150960.

4. Parker R. Construindo os alicerces para a resposta ao HIV/Aids no Brasil: o desenvolvimento de políticas sobre o HIV/Aids, 1982-1996. Saúde Debate. 2003;27:8-49.

5. Mattos RA, Terto VJ, Parker R. As estratégias do Banco Mundial e a resposta à AIDS no Brasil. Saúde Debate. 2003;27:81-94. 
6. Teixeira PR. Políticas públicas em AIDS. In: Parker R, editor. Políticas, instituições e AIDS: enfrentando a epidemia no Brasil. Rio de Janeiro: Jorge Zahar/Abia; 1997. p. 43-69.

7. Banco Mundial. Projeto de controle de AIDS e DST II. Brasilia; 2002. (Relatório PID 11512).

8. Brasil. Ministério da Saúde. Portaria n².313 de 19 de dezembro de 2002. Institui Incentivo para estados, Distrito Federal e municípios no âmbito do Programa Nacional de HIV/Aids e outras DST. Diário Oficial da União, Brasília, 20 de março de 2003.

9. São Paulo. Secretaria de Estado da Saúde. Balanço do Plano de Ações e Metas 2003 [mimeo]. São Paulo; 2004.

10. Silva SR. Gestão e sustentabilidade das ações de prevenção em DST/AIDS. In: Paiva V, França-Júnior I, Kalichman AO, editores. Vulnerabilidade e direitos humanos - prevenção e promoção da saúde: planejar fazer, avaliar. Curitiba: Juruá; 2013. p. 287-308.

11. Brasil. Ministério da Saúde. Portaria no 3.276 de 26 de dezembro de 2013. Regulamenta o incentivo financeiro de custeio às ações de vigilância, prevenção e controle das DST/AIDS e Hepatites Virais. Diário Oficial da União, Brasília, 27 de dezembro de 2013.

12. Brasil. Ministério da Saúde. O que é transferência fundo a fundo [Internet]. Brasília: Departamento de DST, Aids e Hepatites Virais; 2017 [citado em 2017 set 5]. Disponível em: http://www.aids.gov.br/pagina/oque-e-transferencia-fundo-fundo

13. Barbosa-Júnior A, Szwarcwald CL, Pascom ARP, Souza PB Jr. Tendências da epidemia de AIDS entre subgrupos sob maior risco no Brasil, 1980-2004. Cad Saude Publica. 2009;25(4):727-37. http://dx.doi. org/10.1590/S0102-311X2009000400003. PMid:19347198.

14. Berkman A, Garcia J, Muñoz-Laboy M, Paiva V, Parker R. A critical analysis of the Brazilian response to HIV/ AIDS: lessons learned for controlling and mitigating the epidemic in developing countries. Am J Public Health. 2005;95(7):1162-72. http://dx.doi.org/10.2105/AJPH.2004.054593. PMid:15933232.

15. Gil AC. Como elaborar projetos de pesquisa. São Paulo: Atlas; 1991.

16. Minayo MCS. Amostragem e saturação em pesquisa qualitativa: consensos e controvérsias. Rev Pesquisa Qualit. 2017;5(7):1-12.

17. Barboza R. Gestão do Programa Estadual DST/AIDS de São Paulo: uma análise do processo de descentralização das ações no período de 1994 a 2003 [dissertação]. São Paulo: Secretaria de Estado da Saúde de São Paulo; 2006.

18. Minayo MCS. O desafio do conhecimento: pesquisa qualitativa em saúde. São Paulo: Hucitec; 2004.

19. Bardin L. Análise de conteúdo. Lisboa: Edições 70; 1979.

20. Dalfior ET, Lima RCD, Andrade MAC. Reflexões sobre análise de implementação de políticas de saúde. Saúde Debate. 2015;39(104):210-25. http://dx.doi.org/10.1590/0103-110420151040201.

21. Mattos RA. As agências internacionais e as políticas de saúde nos anos 90: um panorama geral da oferta de ideias. Cien Saude Colet. 2001;6(2):377-89. http://dx.doi.org/10.1590/S1413-81232001000200008.

22. Galvão J. AIDS no Brasil: a agenda de construção de uma epidemia. Rio de Janeiro: Abia; 2000.

23. World Bank. Adult health in Brazil: adjusting to new challenges. Washington; 1989. (Report $\mathrm{n}^{\circ} 7807$ - BR).

24. Banco Mundial. Brasil: primeiro e segundo projetos de controle de AIDS e DST. Washington; 2004. (Relatório de avaliação de desempenho de projeto).

25. Grangeiro A, Escuder MM, Gianna MC, Castilho EAC, Teixeira PR. Estratégias de descentralização e municipalização da resposta à aids no Brasil: implicações para as secretarias de saúde e organizações não governamentais. Tempus Actas em Saúde Coletiva. 2010;4(2):15-33. http://dx.doi.org/10.18569/tempus. v4i2.789.

26. Paiva V, Pupo LR, Barboza R. O direito à prevenção e os desafios da redução da vulnerabilidade ao HIV no Brasil. Rev Saude Publica. 2006;40(Supl.1):S109-19. http://dx.doi.org/10.1590/S0034-89102006000800015.

27. São Paulo. Secretaria de Estado da Saúde. Planejamento Estratégico do Programa Estadual DST/AIDS 2002-2005 [mimeo]. São Paulo; 2002.

28. Taglietta MFB. Avaliação da implantação do incentivo no âmbito do Programa Nacional de HIV/AIDS e outras DST em dois municípios do estado de São Paulo [dissertação]. Rio de Janeiro: Fundação Oswaldo Cruz. Escola Nacional de Saúde Pública; 2006.

29. Grangeiro A, Escuder MML, Silva SR, Cervantes V, Teixeira PR. Características da resposta à Aids de secretarias de saúde, no contexto da Política de Incentivo do Ministério da Saúde. Saude Soc. 2012;21(4):954-75 http://dx.doi.org/10.1590/S0104-12902012000400014.

30. Fleury SM, Ouverney AM. Gestão de redes: a estratégia de regionalização da política de saúde. Rio de Janeiro: Fundação Getúlio Vargas; 2011. 